\title{
Can patients use family members as non-professional interpreters in consultations?
}

In an emergency, when a patient doesn't speak your language, it can be tempting to ask a colleague or family member to interpret. This, however, comes with its own risks, as three experts tell Abi Rimmer

Abi Rimmer

The BMJ

\section{Interpreters should be neutral}

Sofia Sarfraz, senior clinical fellow in paediatrics and medical education, says, "For patients who don't share your language the gold standard is to use a professional interpreter. We've all, however, used patients' family members or other health professionals as interpreters in consultations. It's hard to see how medicine could function in our multicultural society without them, especially in emergencies. But non-professional interpreters should be used with caution and you should consider the risks.

"Medical interpreters have training and experience, which family members and other health professionals may lack. Interpreters should be neutral and passive, which may prove difficult for family.

"A family member may also give you their own version of events, and their emphasis may skew the whole consultation. It can also be difficult to check the veracity of the interpretation. This could lead to a misdiagnosis.

"Similarly, a family member may find it hard to share difficult or bad news and they may have emotional or cultural reasons to distort your message. Or they may simply be bashful.

"Be aware that over-reliance on minors as translators can cause them harm. This can range from the child being taken out of school to act as an interpreter, to being traumatised by their exposure to a complex medical communication scenario.

"A final point to consider is that using interpreters can alter the dynamic of the consultation and failing to recognise this can degrade the quality of care. With extra people in the consultation we need to ensure that the patient feels heard and that we pick up on their non-verbal cues.

"Taking all this into consideration, my advice would be to use someone who is impartial-ideally a professional interpreter-where possible in your consultations, excluding emergencies. Should this not be possible, keeping the above in mind, you can use family members with caution and only to keep the patient safe, until other arrangements can be made."

\section{It may be appropriate in an emergency}

Joydeep Grover, consultant in emergency medicine and specialist medicolegal adviser for Medical Defence Shield, says, "The GMC's Good Medical Practice guidance expects doctors to "make sure that arrangements are made, where possible, to meet patients' language and communication needs."

"NHS England, in reference to primary care, states that, 'The use of an inappropriately trained (or no) interpreter poses risks for both the patient and healthcare provider.' And further, 'The error rate of untrained interpreters (including family and friends) may make their use more high risk than not having an interpreter at all.'

"Doctors have a responsibility to communicate effectively with their patients while considering timeliness of effective treatment, their disabilities, cultural needs, and human rights. At the same time, they also have a responsibility to ensure confidentiality, information governance, reliability, and safeguarding.

"Ideally, for all planned consultations, every effort should be made to engage the services of a qualified interpreter using local guidelines with allowances being made for the increased time such consultations take. Family members can, of course, stay for the consultation, if appropriate and with consent.

"Not all situations, however, are ideal; in emergencies where immediate patient care is a priority, an available family member may well be the only means of communication and it may well be appropriate to request them to translate. This, however, must be done with consideration given to confidentiality and safeguarding. The patients' best interests must always take centre stage and if in doubt you can take advice from your defence organisation."

\section{Find out what the patient wants}

Emma Saint, from the standards policy team at the GMC, says, "It's so important that patients get all the information they need about their treatment, even when language presents a barrier. Our guidance is clear that doctors should make arrangements to meet patients' communication needs wherever possible. 
"A professional interpreter is always preferable as they provide a degree of assurance around quality, accuracy, and confidentiality.

"If a professional interpreter isn't immediately available, and it's not possible to delay the conversation, a health professional who is fluent in the patient's language may be able to provide the information that the patient needs or wants to make a decision, and to confirm that they have given consent.

"If that's not an option, you can ask a family member but there are a few things to consider. The first is confidentiality. Before involving family in discussions about a patient's care, you should usually check that the patient wants that to happen. This is clearly difficult if the translator is a relative. It's also possible that patients won't disclose all relevant information through a family member because they don't feel comfortable sharing personal details.

"Family members can also be selective with translations based on their own views of the patient's condition or treatment options. And there is the possibility that they don't understand English well enough to communicate complex medical information.

"There are also some circumstances in which it would be inappropriate to involve family members-for example, where there are safeguarding concerns about the patient.

"So, there are reasons to be cautious about using family members. But ultimately the care of the patient comes first so we advise you to use your professional judgment where interpretation services are unavailable.” 